Psychopharmacologia (Berl.) 22, 271-281 (1971)

(C) by Springer-Verlag 1971

\title{
Psychomotor Stimulant Self Administration as a Function of Dosage per Injection in the Rhesus Monkey*
}

\author{
M. C. WILson**, M. HItomi***, and C. R. Schuster ${ }^{+}$ \\ Pharmacology Department, University of Michigan, Medical School, Ann Arbor, \\ Michigan
}

Received January 20, 1971; Final Version May 17, 1971

\begin{abstract}
The relationships between drug dosage per injection and response rate, and drug dosage per injection and total daily drug intake were ascertained in Rhesus monkeys which self-administered cocaine, pipradrol, methylphenidate and phenmetrazine intravenously. The study demonstrated the monkeys would selfadminister all of these compounds over a wide range of dosages. Furthermore, the magnitude of reinforcement, i.e., dosage per injection, and the rate of responding in self-administering these compounds were inversely related. However, total daily drug intake was independent of the dosage per injection over a wide range of dosages. The results indicate that either the subjects can compensate for large changes in unit dosage so that daily drug intake remains stable or that a direct effect of these compounds functions in limiting their self-administration.
\end{abstract}

Key-Words: Self-Administration - Cocaine - Pipradrol - Phenmetrazine Methylphenidate.

One of the most influential variables which functions in initiating and maintaining a given behavior is the magnitude of a reinforcer which is presented following completion of the appropriate response. The relationship between response rate and magnitude of reinforcement has been investigated in rats self-administering cocaine, methamphetamine, and D-amphetamine intravenously (Pickens, et al., 1967; Pickens and Harris, 1968; and Pickens and Thompson, 1968). These investigators found that an inverse relationship existed between infusion dosage and frequency of self-administration. Furthermore, total daily drug intake was independent of infusion dosage over a wide range of dosages.

* This study was supported by NIMH Grant No. 5R 10MH-12084 and by NIMH Grant No. MH-18245-01.

** Presently located in the Department of Pharmacology, School of Pharmacy, University of Mississippi, Oxford, Mississippi.

*** Presently located at the Fujisawa Drug Company, Osaka, Japan.

+ Presently located in the Departments of Psychiatry and Pharmacology, University of Chicago, Chicago, Illinois. 
Deneau and Inoki using Rhesus monkeys which self-administered nicotine intravenously, likewise demonstrated that an inverse relationship existed between frequency of self-administration and infusion dosage. As a consequence, total daily drug intake remained relatively stable when infusion dosage was varied.

The present study was designed to determine if similar relationships exist between infusion or unit dosage and frequency of self-administration and between infusion dosage and total drug intake in Rhesus monkeys self-administering the psychomotor stimulants cocaine, pipradrol, phenmetrazine and methylphenidate intravenously.

\section{Method}

The subject for this study were eight adult Rhesus monkeys which had no prior drug history and which weighed between 3.6 and $5.8 \mathrm{~kg}$. The subjects were housed in individual open-faced experimental cubicles. The lower back wall consisted of a stainless steel panel; the ceiling, sides and remainder of the back wall were made of nontransparent white plastic. The stainless steel panel contained a lever, stimulus light and water spout. Subject restraint was accomplished by a stainless steel harness and arm (Yanagita et al., 1965). The arm was attached to the back wall of the cage and to the harness. This arrangement provided relatively free movement about the cubicle.

After the subjects had adapted to this environmental situation for seven days, they were surgically prepared with a chronic indwelling jugular catheter of siliconized rubber. The catheter was secured in the right internal jugular vein with the tip terminating in the right atrium of the heart. The other end was passed subcutaneously over the shoulder blade and exited the back where it was attached to an 18 gauge needle located on the back of the harness. A second piece of siliconized rubber tubing was attached to this needle and passed through the restraining arm out the back of the cubicle and was connected to an injector unit. Depression of the response lever with at least $100 \mathrm{~g}$ of force during illumination of the stimulus light activated this unit. This resulted in the intravenous administration of a given volume of drug solution or of physiological saline.

After the subjects were returned to the cubicles following catheterization, the stimulus light was illuminated twenty-four hours a day for seven days. Every lever-press made during this period resulted in the administration of $0.2 \mathrm{cc} / \mathrm{kg}$ of body weight of sterile physiologic saline. The duration of the injection was determined by the animal's weight. During an injection cycle, which lasted approximately forty-five seconds, the stimulus light was turned off and lever-presses although recorded 
had no programmed consequence. Each subject's lever-pressing frequency for saline during this period was determined and this served as a control value.

A $1.0 \mathrm{mg} / \mathrm{cc}$ solution of cocaine hydrochloride in sterile physiologic saline was substituted for the saline at the end of this seven-day period. The subjects had unlimited access to cocaine and each leverpress resulted in the intravenous administration of $0.2 \mathrm{mg} / \mathrm{kg}$ of cocaine hydrochloride. Once a subject had initiated increased self-administration behavior as compared to that observed for saline, the drug access period was reduced from twenty-four to four hours daily. This was done to reduce the neurotoxic effects of cocaine which develop within five to seven days if subjects are allowed unlimited drug access. In addition, limited access resulted in stable daily intake of cocaine, The daily four-hour drug access period which was from 11:00 a.m. to 3:00 p.m. was indicated by the illumination of the stimulus light. During the twenty hour period between sessions, the light was never illuminated and lever-presses had no programmed consequence.

After each subject established stable self-administration patterns $(<10 \%$ change in daily drug intake) for cocaine, the cocaine solution was replaced with a solution of either methylphenidate hydrochloride, pipradol hydrochloride, phenmetrazine hydrochloride or cocaine hydrochloride of a different concentration. The volume of each injection remained constant throughout the study; all drugs were dissolved in physiologic saline and dosages calculated on the basis of the salt. Only the concentrations of the drug solution changed during the study. New solutions were prepared every third day, and old solutions were discarded.

Subjects were maintained at each unit dosage (dosage per injection) level for seven consecutive days. The unit dosage was unsystematically varied over a wide range which, for cocaine, was $0.025-1.2 \mathrm{mg} / \mathrm{kg}$; phenmetrazine, $0.025-0.8 \mathrm{mg} / \mathrm{kg}$; methylphenidate, $0.025-0.4 \mathrm{mg} / \mathrm{kg}$ and pipradrol, $0.025-0.4 \mathrm{mg} / \mathrm{kg}$.

The effects of varying the unit dosage, i.e., magnitude of reinforcement of cocaine and pipradrol was studied in four subjects and of phenmetrazine and methylphenidate in four other subjects. Every subject was exposed to each unit dosage of the compound being tested and at least five unit dosages of each drug were studied. Studies with one compound were completed before a second compound was tested in a given subject.

\section{Results}

Fig. 1 illustrates the mean number of responses per session (i.e., the frequency of self-administration) and mean drug intake per session as functions of the unit dosage of cocaine hydrochloride. Each point 


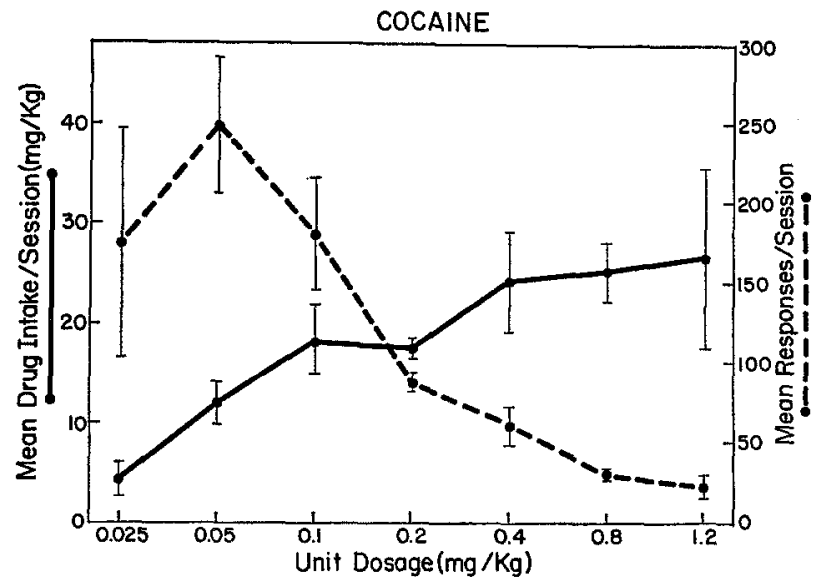

Fig. 1. Mean number of responses per session (broken line) and mean drug intake per session (solid line) as functions of unit dosage of cocaine hydrochloride. Each point represents the mean of data obtained from four subjects on days three through seven of exposure to each unit dosage. Vertical lines represent the range

\section{COCAINE}

25

50

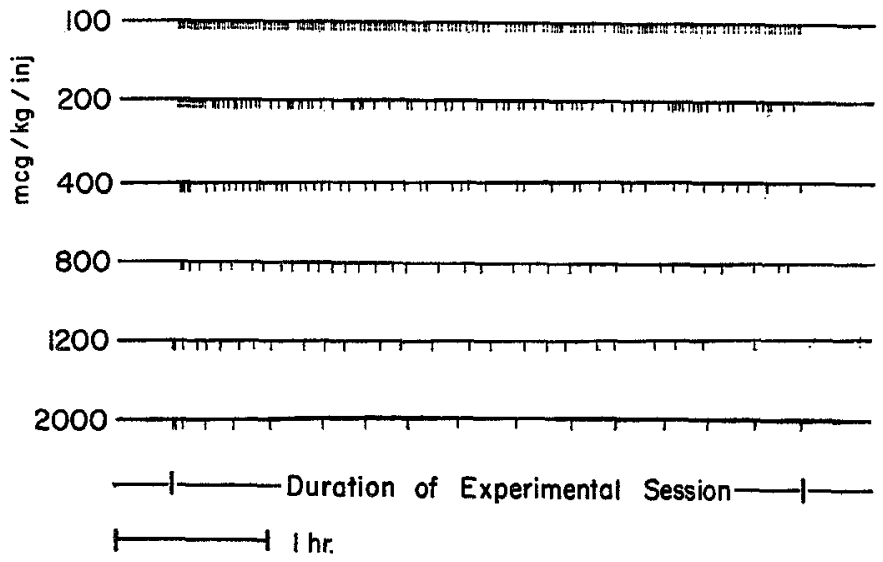

Fig. 2. Selected session records at each unit dosage of cocaine studied in one subject. Each vertical line represents one response or one self-administration 
represents the mean of data obtained from four subjects on days three through seven at each unit dosage level. The vertical lines represent the range. As the unit dosage was increased from $0.05 \mathrm{mg} / \mathrm{kg}$ to $1.2 \mathrm{mg} / \mathrm{kg}$, the mean number of responses emitted, i. e., injections received, decreased from 250 to 5 . Mean drug intake per session increased from 4.5 to $18.0 \mathrm{mg} /$ $\mathrm{kg}$ as the unit dosage was increased from 0.025 to $0.1 \mathrm{mg} / \mathrm{kg}$.

Further increments in unit dosage produced only small increases in drug intake as the unit dosage was increased from 0.1 to $1.2 \mathrm{mg} / \mathrm{kg}$. Mean drug intake increased only from 18.1 to $24.8 \mathrm{mg} / \mathrm{kg}$.

At a unit dosage of $0.025 \mathrm{mg} / \mathrm{kg}$ significant variability in responding occurred both between animals and in the same animals from one day to the next. This is probably attributable to the fact that this dosage is at the threshold value for reinforcement.

Limitations of the equipment prevented the subjects from selfadministering a total dosage of cocaine at a unit dosage of $0.05 \mathrm{mg} / \mathrm{kg}$ as was self-administered at higher unit dosages. Each injection cycle lasted from 45-60 sec, depending on the weight of the subject. Therefore, approximately only 300 injections could be self-administered during the four hour session.

The total daily intake of cocaine self-administered by these subjects appears to be independent of unit dosage, i.e., magnitude of reinforcement, over a range which maintained self-administration behavior. Even though unit dosage was increased by a factor of twelve, i.e., from 0.1 to $1.2 \mathrm{mg} / \mathrm{kg}$, mean daily drug intake increased by only a factor of two due to the inverse relationship between unit dosage and response rate over this range.

Fig. 2 illustrates a selected session record at each unit dosage of cocaine studied in one subject. Each vertical line represents a single response or self-administration. At unit dosages of 0.025 and $0.05 \mathrm{mg} / \mathrm{kg}$, the infusion pump was almost constantly operating throughout the session. As the unit dosage was increased from 0.1 to $2.0 \mathrm{mg} / \mathrm{kg}$, the number of lever-presses decreased. Following an initial burst of responding, the subjects responded at a fairly constant rate throughout the remainder of the session. A characteristic pause between responding occurred at each unit dosage level. The duration of this pause increased in length and became more regular as the unit dosage was increased.

Similar results were obtained with pipradrol self-administration. Fig. 3 illustrates the mean number of responses per session and mean drug intake per session as functions of the unit dosage of pipradrol hydrochloride. Pipradrol was self-administered at unit dosage levels of $0.025-0.4 \mathrm{mg} / \mathrm{kg}$. As the unit dosage was increased from 0.05 to $0.4 \mathrm{mg} /$ $\mathrm{kg}$, the mean number of injections per session decreased from 118 to 27 . The large variability in responding seen at a unit dosage of $0.025 \mathrm{mg} / \mathrm{kg}$ 


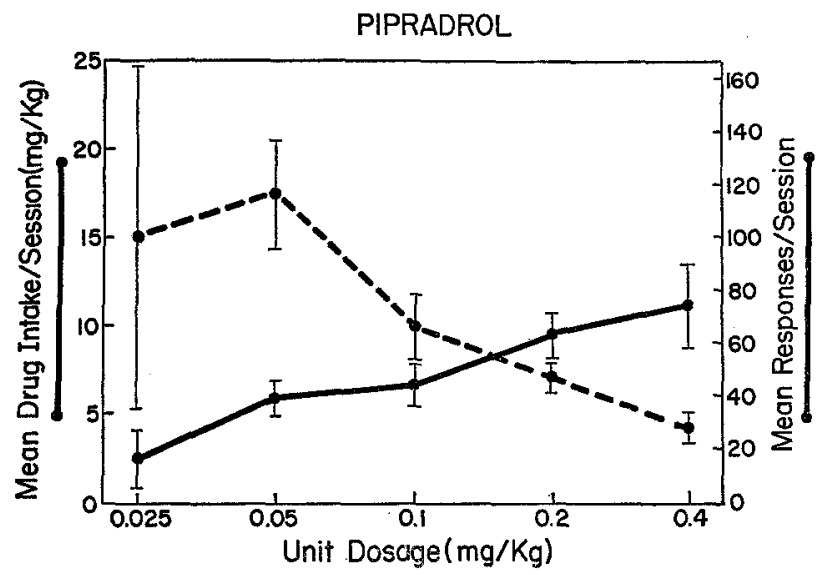

Fig. 3. Mean number of responses per session (broken line) and mean drug intake per session (solid line) as functions of unit dosage of pipradrol hydrochloride. Each point represents the mean of data obtained from four subjects on days three through seven of exposure to each unit dosage. Vertical lines represent the range

\section{PIPRADROL}

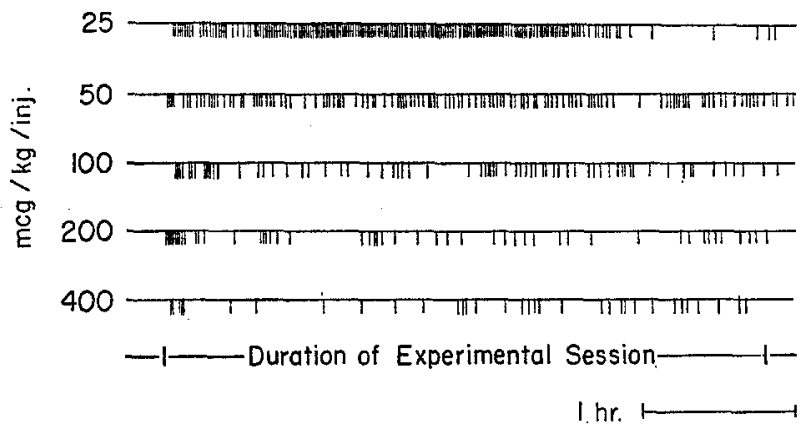

Fig.4. Selected session records at each unit dosage of pipradrol studied in one subject. Each vertical line represents one response or one self-administration

is probably attributable to intersubject variability in the threshold reinforcing dose of pipradrol. Response rate variability decreased as the unit dosage increased. Mean drug intake per session increased from 5.8 to $11.2 \mathrm{mg} / \mathrm{kg}$ as unit dosage was increased from 0.05 to $0.4 \mathrm{mg} / \mathrm{kg}$. Therefore, there was only a twofold increase in drug intake produced by an eightfold increase in unit dosage. As occurred with cocaine, response rate was inversely proportional to unit dosage and total drug intake was relatively independent of dosage at unit dosage levels shown to be reinforcing. 


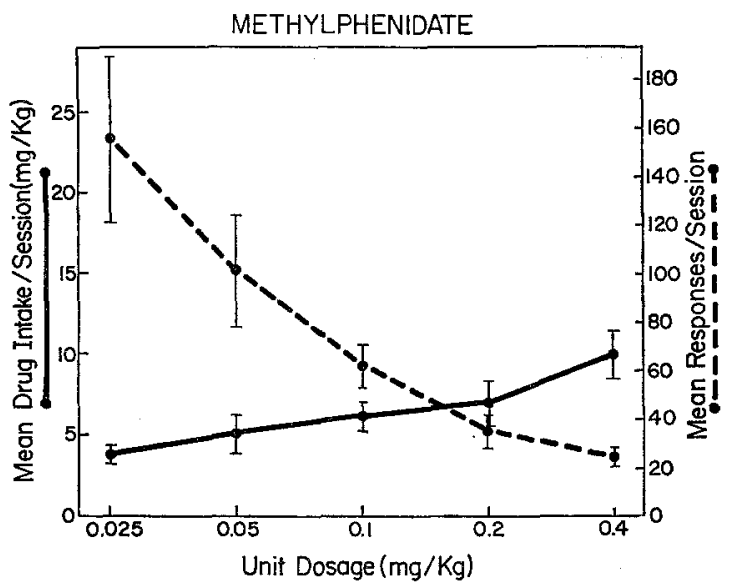

Fig. 5. Mean number of responses per session (broken line) and mean drug intake per session (solid line) as functions of unit dosage of methylphenidate hydrochloride. Each point represents the mean of data obtained from four subjects on days three through seven of exposure to each unit dosage. Vertical lines represent the range

\section{METHYLPHENIDATE}

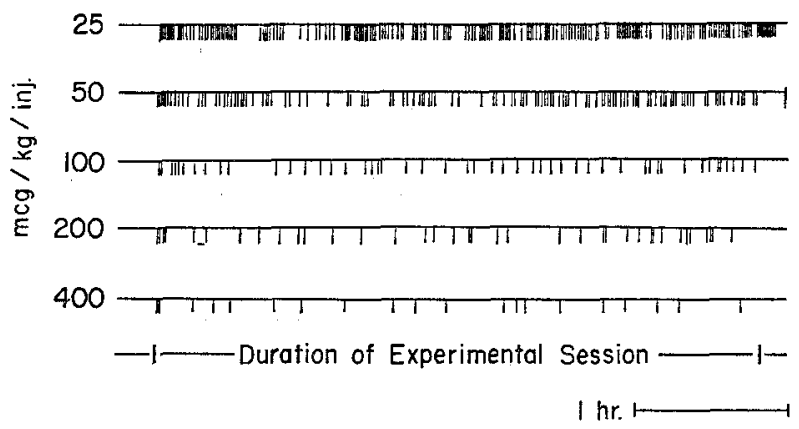

Fig.6. Selected session records at each unit dosage of methylphenidate studied in one subject. Each vertical line represents one response or one self-administration

Fig. 4 illustrates a selected session record at each unit dosage level of pipradrol studied in one subject. As the unit dosage of pipradrol was increased, response rate decreased and the length of the post-reinforcement pause increased and became more uniform in length. Response rate at each unit dosage was fairly constant throughout the duration of the session; however, as with cocaine, the rate at the onset of the session was higher than at any other time during the session.

Response rate and unit dosage were also inversely related in subjects self-administering methylphenidate. Fig.5 illustrates the mean number 


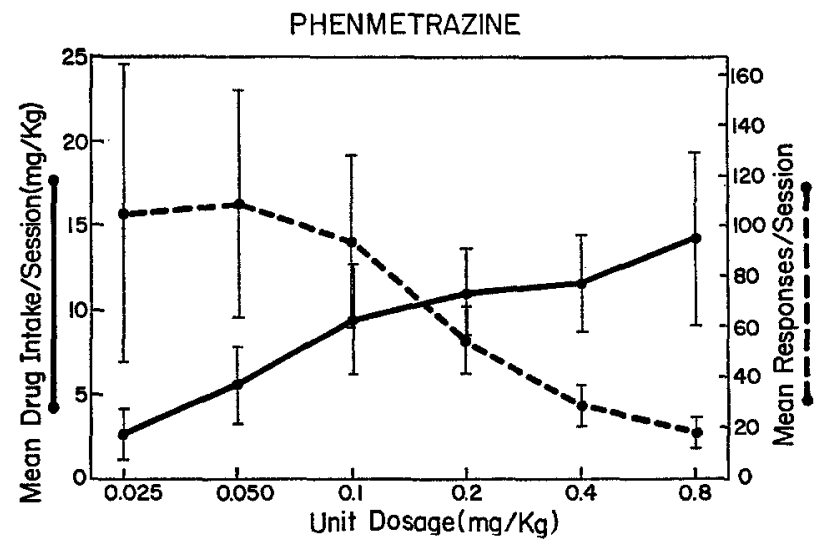

Fig. 7. Mean number of responses per session (broken line) and mean drug intake per session (solid line) as functions of unit dosage of phenmetrazine hydrochloride. Each point represents the mean of data obtained from four subjects on days three through seven of exposure to each unit dosage. Vertical lines represent the range

\section{PHENMETRAZINE}

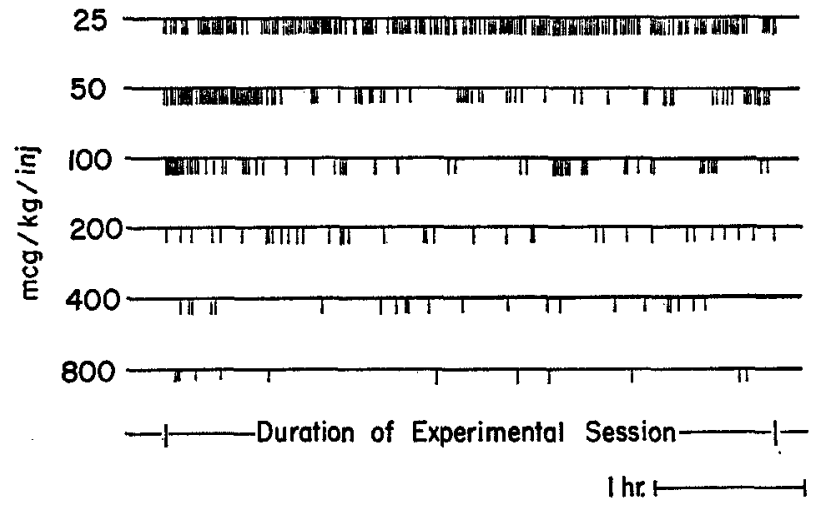

Fig. 8. Selected session records at each unit dosage of phenmetrazine studied in one subject. Each vertical line represents one response or one self-administration.

of responses per session and mean drug intake per session as functions of the unit dosage of methylphenidate hydrochloride. As unit dosage increased from 0.025 to $0.4 \mathrm{mg} / \mathrm{kg}$, mean responses per session decreased from 159 to 24 . Variability in responding was greatest at the lowest unit dosage and decreased as unit dosage was increased. Mean drug intake per session increased from 4.1 to $8.7 \mathrm{mg} / \mathrm{kg}$ over this range of unit dosage. Therefore, drug intake only doubled while unit dosage was increased by a factor of sixteen. 
Fig. 6 illustrates session records obtained from a subject at each unit dosage of methylphenidate studied. Increases in unit dosage produced a decrease in responding. Response rate at a given unit dosage, except for an initial higher rate at the start of the session, was fairly constant throughout the remainder of the session. The post-reinforcement pause in responding became longer and more uniform in duration with increases in unit dosage.

The results obtained with phenmetrazine self-administration were very comparable to those obtained with cocaine, pipradrol and methylphenidate. Fig. 7 shows the mean number of responses per session and mean drug intake per session, as functions of the unit dosage of phenmetrazine hydrochloride. As the unit dosage was increased from 0.05 to $0.8 \mathrm{mg} / \mathrm{kg}$. the mean number of responses per session decreased from 108 to 18. Mean drug intake per session over this same range of unit dosage increased from 5.2 to $14.6 \mathrm{mg} / \mathrm{kg}$. Therefore, although unit dosage had been increased by a factor of sixteen, there was only a threefold increase in drug intake. As occurred with the other psychomotor stimulants studied, variability in responding was greatest at the lowest unit dosage and this variability decreased with increases in unit dosage.

Fig. 8 illustrates selected session records at each unit dosage of phenmetrazine studied in one subject. As unit dosage increased, the length of pausing after reinforcement, although regular, increased and response rate decreased. Response rates were constant at a given unit dosage throughout the session following an initial burst of responding at the beginning of the session.

\section{Discussion}

The results of this study demonstrate that an inverse relationship exists between rate of responding and the magnitude of reinforcement, i.e., unit dosage, in Rhesus monkeys self-administering cocaine, methylphenidate, pipradrol and phenmetrazine. A similar inverse relationship between magnitude of reinforcement and response rate has been demonstrated in rats self-administering cocaine, methamphetamine and d-amphetamine (Pickens and Thompson, 1968; Pickens et al., 1967; Pickens and Harris, 1968), and in monkeys self-administering nicotine (Deneau and Inoki).

However, across a wide range of unit dosages daily drug intake is independent of magnitude of reinforcement. It has also been demonstrated by Woods and Schuster that tne mean daily intake of cocaine in Rhesus monkeys is very stable at a given unit dosage, i.e., variability never exceeded $\pm 10 \%$. In addition, total intake remained constant across dosages. 
Their data suggests that some mechanism is involved in regulating or limiting drug intake. The results of this present study also tend to implicate such a system.

Pickens and Thompson (1968) have demonstrated in rats selfadministering cocaine that total daily drug intake was independent of the magnitude of reinforcement. These authors demonstrated that the same relationship exists in rats working for food pellets.

One may say that this data imply that the subjects self-administering psychomotor stimulants adjust their response rates to maintain an optimum blood level of these compounds in much the same manner that central and peripheral mechanisms may regulate or control the selfadministration of other reinforcers, e.g. food and water. However, we do not have any data related to the actual blood levels of these compounds during the drug access period. Perhaps even though total drug intake may be constant, actual blood levels obtained may be different.

Another possible explanation of these results is that after a given amount of psychomotor stimulant is administered, all on-going behavior or reinforcement-seeking is suppressed. It has been frequently demonstrated that large doses of psychomotor stimulants decrease responding in a variety of behavioral tasks in which other types of reinforcers were used. Pickens and Thompson (1968) demonstrated that an intravenous infusion of cocaine completely disrupted lever-pressing behavior in rats working for food reinforcement. The duration of this disruption was directly related to the amount of cocaine infused. Whether the result was due to a specific anorexic effect or to a general behavioral disrupting effect of cocaine could not be definitely determined.

The pattern of responding for psychomotor stimulants by the monkeys supports this explanation. The initial burst of responding at the onset of the session may result in the administration of a sufficient quantity of drug which interferes with additional drug-seeking behavior. As soon as some of the drug is metabolized. redistributed, etc., the subject again initiates lever-pressing behavior. This cycle would then be repeated throughout the duration of the session. As the unit dosage was increased, the length of the pause after each injection increased. This may have occurred because more time was required for the drug present in each injection to be inactivated.

A third possible explanation for these results is that these compounds produce aversive effects when a sufficient amount has been self-administered. Further injections would be more aversive so the subject stops responding until a sufficient amount of the drug is metabolized, thereby reducing the aversiveness. The subjects could now self-administer more of the compound until this aversive level is again reached. This cycle would then be repeated throughout the drug-access period. 
There is evidence that large dosages of the psychomotor stimulants do produce aversive effects. Many cocaine abusers have self-administered heroin in an effort to overcome or prevent the occurence of this effect (Goodman and Gilman).

Plutchik et al., have shown that in rats there is an increase in selfstimulation rates with increases in current intensity up to a maximum. With additional increases in current intensity, self-stimulation behavior decreases. It has been speculated that with increasing intensity of stimulation, "punishment" areas of the brain in close proximity to these "reward" areas become stimulated, thereby decreasing the overall reinforcing effects of brain stimulation. This may be analogous to the hypothesis that aversive effects are produced by psychomotor stimulants when a sufficient quantity is administered, and it is these aversive effects which limit the amount of psychomotor stimulants which are self-administered.

Further studies must be carried out in an effort to determine what the exact nature of the mechanism is which functions to limit the selfadministration of these psychomotor stimulants in rats and Rhesus monkeys.

\section{References}

Deneau, G., Inoki, R.: Nicotine self-administration in monkeys. Ann. N. Y. Acad. Sci. 142, 277-279 (1967).

Goodman, L., Gilman, A.: The pharmacological basis of therapeutics. 4th Edition. New York: MacMillan 1970.

Pickens, R., Harris, W.: Self-administration of d-amphetamine by rats. Psychopharmacologia (Berl.) 12, 158-163 (1968).

- Meisch, R., McQuire, L.: Methamphetamine reinforcement in rats. Psychonom. Sci. 8, 371-372 (1967).

- Thompson, T.: Cocaine-reinforced behavior in rats: Effects of reinforcement magnitude and fixed ratio size. J. Pharmacol. exp. Ther. 161, 122-129 (1968).

Plutchik, R., McFarland, W., Robinson, B.: Relationships between current intensity, self-stimulation rates, escape latencies and evoked behavior in Rhesus monkeys. J. comp. physiol. Psychol. 61, 181-188 (1966).

Woods, J., Schuster, C. R.: Reinforcement properties of morphine, cocaine and SPA as a function of unit dose. Int. J. Addict. 3, 231-237 (1968).

Yanagita, T., Deneau, G., Seevers, M.: Evaluation of pharmacological agents in the monkey by long-term intravenous self or programmed administration. Excerpta Medica International Congress Series 87, 453-457 (1965).

Dr. C. R. Schuster

Department of Psychiatry

University of Chicago

950 East 59th Street

Chicago, Illinois 60637

U.S.A. 